\title{
Compressibility of rotating black holes
}

\author{
Brian P. Dolan \\ Department of Mathematical Physics, National University of Ireland, Maynooth, Ireland \\ and Dublin Institute for Advanced Studies, 10 Burlington Road, Dublin, Ireland
}

(Received 2 September 2011; published 16 December 2011)

\begin{abstract}
Interpreting the cosmological constant as a pressure, whose thermodynamically conjugate variable is a volume, modifies the first law of black hole thermodynamics. Properties of the resulting thermodynamic volume are investigated: the compressibility and the speed of sound of the black hole are derived in the case of nonpositive cosmological constant. The adiabatic compressibility vanishes for a nonrotating black hole and is maximal in the extremal case - comparable with, but still less than, that of a cold neutron star. A speed of sound $v_{s}$ is associated with the adiabatic compressibility, which is equal to $c$ for a nonrotating black hole and decreases as the angular momentum is increased. An extremal black hole has $v_{s}^{2}=0.9 c^{2}$ when the cosmological constant vanishes, and more generally $v_{s}$ is bounded below by $c / \sqrt{2}$.
\end{abstract}

DOI: 10.1103/PhysRevD.84.127503

PACS numbers: 04.70.Dy

\section{INTRODUCTION}

It has been suggested by a number of authors [1-8] that, for a black hole of mass $M$ when a cosmological constant is included, the first law of thermodynamics should read

$$
d M=T d S+\Omega d J+\Phi d Q+\Theta d \Lambda,
$$

where $S, J$, and $Q$ are the entropy, angular momentum, and charge, respectively, while $\Theta$ is a thermodynamic variable conjugate to the cosmological constant $\Lambda$. Since $\Lambda$ is associated with a pressure, $\Lambda=-8 \pi P$ in units with $G=$ $c=1$, one interpretation of the $\Theta d \Lambda$ term is that it is related to the familiar term $P d V$ term in the first law, with $\Theta$ proportional to the volume. This in turn requires interpreting the black hole mass as corresponding to the thermodynamic enthalpy, $H=M(S, P, J, Q)$, rather than the more usual interpretation as the thermal energy $U$ $[9,10]$. One then has

$$
d M=T d S+\Omega d J+\Phi d Q+V d P,
$$

where the thermodynamic volume,

$$
V=\left.\frac{\partial M}{\partial P}\right|_{S, J, Q}=-8 \pi \Theta
$$

is the Legendre transform of the pressure. In full generality, the first law of black hole thermodynamics now reads

$$
d U=T d S+\Omega d J+\Phi d Q-P d V,
$$

where $U=H-P V$. In this work we shall set $Q=0$ for simplicity; our results are easily extended to include the $Q \neq 0$ case.

Of course if $\Lambda$ is truly constant, $P$ cannot be varied and the last term in (2) is always zero, though the last term in (4) need not be zero and can have physical consequences [11]. But it is commonly supposed, in inflationary models, for example, that $\Lambda$ has at some time in the past been

\footnotetext{
*bdolan@thphys.nuim.ie
}

significantly different from its current value and so must vary.

Unfortunately the thermodynamics of black holes with $\Lambda>0$ are not well understood: such a system has two different temperatures in general, one associated with the black hole horizon and one with the cosmological horizon, and in addition there is no asymptotic regime in which an invariant mass can be defined. For these reasons we shall primarily restrict ourselves to the better understood $\Lambda<0$ case in this work. This is sufficient to access the $\Lambda=0$ limit.

The thermodynamics of anti-de Sitter black holes was examined in detail in the seminal paper of Hawking and Page [7] and there has been a large body of work on this topic since then. The mass of a charged AdS-Kerr black hole, with cosmological constant $\Lambda=-8 \pi P$, was expressed as a function of $S, J$, and $Q$ in [8]. When $Q=0$ the expression is

$$
M(S, P, J)=\frac{1}{2} \sqrt{\frac{\left(1+\frac{8 P S}{3}\right)\left\{S^{2}\left(1+\frac{8 P S}{3}\right)+4 \pi^{2} J^{2}\right\}}{\pi S}},
$$

where $G=c=1$ and $S$ is $\frac{1}{4}$ the area of the event horizon. From this the temperature can be evaluated,

$T=\left.\frac{\partial M}{\partial S}\right|_{P, J}=\frac{1}{8 \pi M}\left\{\left(1+\frac{8 P S}{3}\right)(1+8 P S)-4 \pi^{2} \frac{J^{2}}{S^{2}}\right\}$,

with $\hbar=1$. The temperature vanishes for extremal black holes, with maximum angular momentum

$$
J_{\max }^{2}=\left(\frac{S}{2 \pi}\right)^{2}\left(1+\frac{8 P S}{3}\right)(1+8 P S) .
$$

The stability properties of Kerr-AdS black holes were studied in [8], where it was shown that there is a critical point near $J=0.0236$ (the authors of [8] used units in which $P=\frac{3}{8 \pi}$ ). 
The thermodynamic volume (3),

$$
V=\left.\frac{\partial M}{\partial P}\right|_{S, J}=\frac{2}{3 \pi M}\left\{S^{2}\left(1+\frac{8 P S}{3}\right)+2 \pi^{2} J^{2}\right\}
$$

is always greater than the naive geometric result $V=\frac{4 \pi}{3}\left(\frac{S}{\pi}\right)^{3 / 2}$, as observed in [12].

The Legendre transform of (5),

$U(S, V, J)=\left(\frac{\pi}{S}\right)^{3}\left\{\left(\frac{3 V}{4 \pi}\right)\left(\frac{S^{2}}{2 \pi^{2}}+J^{2}\right)-J^{2} \sqrt{\left(\frac{3 V}{4 \pi}\right)^{2}-\left(\frac{S}{\pi}\right)^{3}}\right\}$,

was studied in [11], where the difference between the specific heat at constant pressure

$$
C_{P}=\left.T \frac{\partial S}{\partial T}\right|_{P, J}=T \frac{1}{\left.\frac{\partial^{2} H}{\partial S^{2}}\right|_{P, J}}
$$

and specific heat at constant volume

$$
C_{V}=\left.T \frac{\partial S}{\partial T}\right|_{V, J}=T \frac{1}{\left.\frac{\partial^{2} U}{\partial S^{2}}\right|_{V, J}}
$$

was also explored. Explicit expressions confirm that $\frac{C_{P}}{C_{V}} \geq 1$. $C_{P}$ diverges along the curve in the $J-S$ plane shown in Fig. 1 (plotted using dimensionless variables $J P$ and $S P) . C_{P}$ is negative in the region below this curve and the critical point corresponds to the maximum value of $J P$ which is determined numerically to lie at $J P=0.00286 \ldots$ and $S P=0.0820 \ldots$ When $J=0$,

$$
C_{P}=\frac{2 S(1+8 P S)}{8 P S-1}
$$

which is negative when $P=0$, the famous negative heat capacity of Schwarzschild black holes refers to $C_{P}$. This instability is reflected in the isothermal compressibility

$$
\beta_{T}=-\frac{1}{V}\left(\frac{\partial V}{\partial P}\right)_{T, J}=\frac{48 S\left\{9 j^{6}+3(5+8 p)(3+8 p) j^{4}+6(3+8 p)^{3} j^{2}+(3+8 p)^{4}\right\}}{\left(6+16 p+3 j^{2}\right)\left\{9(9+32 p) j^{4}+6(3+16 p)(3+8 p)^{2} j^{2}+(8 p-1)(3+8 p)^{3}\right\}},
$$

where $j=2 \pi J / S$ and $p=P S . \beta_{T}$ diverges when the denominator vanishes, which is the same locus of points on which $C_{P}$ diverges and is shown below in the $J P-S P$ plane appropriate for constant $P$ processes.

For a nonrotating black hole

$$
\left.\beta_{T}\right|_{J=0}=\frac{24 S}{8 P S-1}
$$

is negative for $P S<\frac{1}{8}$.

Perhaps a more useful quantity to consider is the adiabatic compressibility,

$$
\beta_{S}=-\frac{1}{V}\left(\frac{\partial V}{\partial P}\right)_{S, J} .
$$

Evaluating $\beta_{S}$ from (8) shows that it is manifestly positive and regular,

$$
\beta_{S}=\frac{36 S j^{4}}{(3+8 p)\left(3+8 p+3 j^{2}\right)\left(6+16 p+3 j^{2}\right)} .
$$

Thus increasing the pressure, keeping the area and angular momentum constant, causes the volume of the black hole to decrease, while at the same time the mass must increase according to (5). $\beta_{S}$ vanishes when $J=0$, so nonrotating black holes are adiabatically incompressible, but it increases with $J$ attaining a maximum value in the extremal case (7) when

$$
\left.\beta_{S}\right|_{T=0}=\frac{2 S(1+8 p)^{2}}{(3+8 p)^{2}(1+4 p)} .
$$

A speed of sound, $v_{s}$, can also be associated with the black hole, in the usual thermodynamic sense that

$$
v_{s}^{-2}=\left.\frac{\partial \rho}{\partial P}\right|_{S, J}=1+\rho \beta_{S}=1+\frac{9 j^{4}}{\left(6+16 p+3 j^{2}\right)^{2}},
$$

where $\rho=\frac{M}{V}$ is a density. This is not the sound associated with any kind of surface wave on the event horizon, an impossibility due to the no-hair theorem, but rather it is associated with a breathing mode for the black hole as the volume changes with the pressure, keeping the area constant. Clearly $0 \leq v_{s}^{2} \leq 1$ (this is also true when $Q \neq 0$ ). The speed of sound is unity for incompressible nonrotating black holes and is lowest for the extremal case

$$
\left.v_{s}^{-2}\right|_{T=0}=1+\left(\frac{1+8 p}{3+8 p}\right)^{2},
$$

giving $v_{s}^{2}=0.9$ when $P=0$ and $v_{s}^{2}$ achieving a minimum value of $\frac{1}{2}$ when $P S \rightarrow \infty$.

These results show that the equation of state is very stiff for adiabatic variations of nonrotating black holes and softens as $J$ increases. For comparison, the adiabatic compressibility of a degenerate gas of $N$ relativistic neutrons in a volume $V$ at zero temperature follows from the degeneracy pressure

$$
P_{\mathrm{deg}}=\left(3 \pi^{2}\right)^{1 / 3} \frac{c \hbar}{4}\left(\frac{V}{N}\right)^{-4 / 3} \Rightarrow \beta_{S}=\frac{3}{4 P_{\mathrm{deg}}} .
$$

For a neutron $\operatorname{star} \frac{N}{V} \approx 10^{45} \mathrm{~m}^{-3}$ and $\beta_{S} \approx 10^{-34} \mathrm{kgms}^{-2}$. With zero cosmological constant the black hole adiabatic compressibility at zero temperature is given by (17) with $P=0$, 


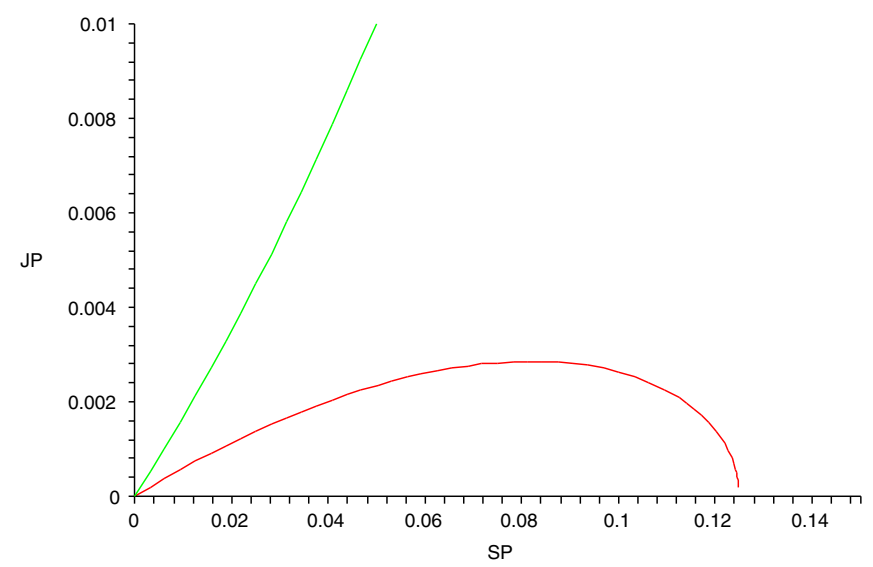

FIG. 1 (color online). Region in the $J P-S P$ plane in which $C_{P}$ is negative. $C_{P}$ diverges on the lower curve and is negative below it. The upper curve shows $J_{\max }$, i.e. $T=0$, on which $C_{P}$ vanishes.

$$
\left.\beta_{S}\right|_{T=P=0}=\frac{2 S}{9}=\frac{4 \pi M^{2} G^{3}}{9 c^{8}}
$$

where the relevant factors of $c$ and $G$ are included. Putting in the numbers

$$
\left.\beta_{S}\right|_{T=0}=2.6 \times 10^{-38}\left(\frac{M}{M_{\odot}}\right)^{2} \mathrm{~m} \mathrm{~s}^{2} \mathrm{~kg}^{-1},
$$

which is still 2 orders of magnitude less than that of a neutron star even for $M \approx 10 M_{\odot}$. We conclude that the zero temperature black hole equation of state is stiffer than that of a neutron star.

A subtlety with this analysis of adiabatic compressibility is that, if a black hole is extremal and the pressure goes down, keeping the entropy and the angular momentum fixed, then the extremal value of the angular momentum
(7) goes down and the black hole ends up with an angular momentum above the new extremal value, giving a naked singularity. One interpretation of this would be that cosmic censorship simply does not allow such a process to occur and, at zero $T$, the entropy must increase, if the pressure is decreased, in such a way as to avoid the extremal angular momentum going down. If the extremal angular momentum is kept constant then, from (7),

$$
\left.\frac{\partial S}{\partial P}\right|_{T=0}=-\frac{16 S^{2}(1+4 p)}{3+48 p+128 p^{2}} .
$$

With this constraint the zero temperature compressibility is increased above the adiabatic value; in fact it becomes identical to the zero temperature isothermal compressibility,

$$
\beta_{T=0}=\frac{2 S\left(11+80 p+128 p^{2}\right)}{(1+4 p)\left(3+48 p+128 p^{2}\right)} .
$$

At zero pressure this is a factor of 33 larger than (21), hence cosmic censorship effectively makes the zero temperature equation of state softer.

Although the analysis presented here is strictly only valid for $P \geq 0$ nothing obviously goes wrong for $P<0$, provided $P S \geq-\frac{1}{8}$, and it may be reasonable to analytically continue these thermodynamic relations to negative pressures [13], provided the resulting positive cosmological constant is not too large.

It has been suggested that mini black holes could have been created in the early Universe. If this is the case, and they are created with a range of angular momenta, it is interesting to speculate that a collection of such compressible black holes might affect the speed of sound through the surrounding medium, in the same way that a suspension of compressible spheres would affect the speed of sound in a fluid.
[1] M. Henneaux and C. Teitelboim, Phys. Lett. 143B, 415 (1984); 222, 195 (1989).

[2] C. Teitelboim, Phys. Lett. 158B, 293 (1985).

[3] S. Wang, S.-Q. Wu, F. Xie, and L. Dan, Chin. Phys. Lett. 23, 1096 (2006).

[4] Y. Sekiwa, Phys. Rev. D 73, 084009 (2006).

[5] E. A. Larrañaga Rubio, arXiv:0711.0012.

[6] S. Wang, arXiv:gr-qc/0606109.

[7] S. W. Hawking and D. N. Page, Commun. Math. Phys. 87, 577 (1983).
[8] M. M. Caldarelli, G. Cognola, and D. Klemm, Classical Quantum Gravity 17, 399 (2000).

[9] D. Kastor, S. Ray, and J. Traschen, Classical Quantum Gravity 26, 195011 (2009).

[10] B.P. Dolan, Classical Quantum Gravity 28, 125020 (2011).

[11] B.P. Dolan, Classical Quantum Gravity 28, 235017 (2011).

[12] M. Cvetic, G. W. Gibbons, D. Kubiznak, and C. N. Pope, Phys. Rev. D 84, 024037 (2011).

[13] B. Lukács and K. Martinás, Acta Phys. Pol. B 21, 177 (1990). 